\title{
Quantitative pixel-wise measurement of myocardial blood flow: a comparison of methods to correct for surface coil-related field inhomogeneity
}

\author{
Christopher A Miller*, Li-Yueh Hsu, Allison D Ta, Hannah Conn, Susanne Winkler, Andrew E Arai
}

From 17th Annual SCMR Scientific Sessions

New Orleans, LA, USA. 16-19 January 2014

\section{Background}

Pixel-wise quantitative analysis of cardiovascular magnetic resonance (CMR) perfusion images allows myocardial blood flow (MBF) to be measured at the level of approximately $30 \mu \mathrm{L}$ of myocardium. However B1-field inhomogeneity, induced by phased array surface receiver coils, potentially confounds MBF measurements. The aim of this study was to compare the effect of: 1 . no surface coil intensity correction (No-SCIC); 2. SCIC performed using saturation recovery steady-state free precession images (SSFP-SCIC); and 3. SCIC performed using proton density-weighted images (PD-SCIC); on pixel-wise measurements of MBF in patients with confirmed significant coronary artery disease (CAD) and healthy volunteers.

\section{Methods}

26 subjects, comprising 18 patients (11 male; age $58 \pm$ 12 years) with significant CAD ( $>70 \%$ stenosis on invasive coronary angiography) in $1(56 \%)$ or $2(44 \%)$ major epicardial arteries (78\% LAD, 50\% RCA, 17\% Cx) and 8 healthy volunteers ( 7 male; age $24 \pm 10$ years), were included. Perfusion imaging was performed using a motion-corrected saturation recovery steady-state free precession, dual sequence method at 1.5T (Espree, Siemens Medical Solutions) during Regadenoson vasodilator stress and at rest, using $0.05 \mathrm{mmol} / \mathrm{kg}$ Gadolinium-DTPA. A proton density-weighted image was acquired at the beginning of the perfusion sequence. B1-field inhomogeneity was approximated using a third-order surface fit to myocardial and body signal intensities on the PD image or the first SSFP image. The estimated intensity bias field was

\footnotetext{
Cardiovascular and Pulmonary Branch, National Heart, Lung and Blood
} Institute, National Institutes of Health, Bethesda, Maryland, USA subsequently applied to the image series. For No-SCIC analysis, only baseline normalisation was performed. Pixelwise MBF was measured from mid-ventricular stress images.

\section{Results}

There was significantly greater variation in signal intensity on the corrected SSFP-SCIC images (coefficient of variation $20 \pm 7 \%)$ compared to the PD-SCIC images $(10 \pm 5 \%$; $\mathrm{p}<0.001$, Figure 1 ). There was also significantly greater MBF spatial heterogeneity (standard deviation divided by mean) in healthy volunteers using SSFP-SCIC (24.8 \pm $4.1 \%)$ compared to PD-SCIC $(20.8 \pm 3.0 \%$; $\mathrm{p}=0.009)$. Using No-SCIC, spatial heterogeneity was substantially higher $(36.2 \pm 6.3 \%$; $p=0.001$ compared with SSFP-SCIC; $\mathrm{p}<0.001$ compared with PD-SCIC). However, the difference in MBF between remote and ischaemic territories (using standard AHA/ACC segmentation) was not significantly different for SSFP-SCIC $(0.63 \pm 0.89 \mathrm{~mL} / \mathrm{min} / \mathrm{kg})$ compared to $\mathrm{PD}$-SCIC $(0.50 \pm 0.63 \mathrm{~mL} / \mathrm{min} / \mathrm{kg} ; \mathrm{p}=$ $0.145)$. Using No-SCIC, the difference in MBF between remote and ischaemic territories was minimal $(0.06 \pm 0.91$ $\mathrm{mL} / \mathrm{min} / \mathrm{kg}$ ), and significantly lower than for both $\operatorname{SSFP-SCIC~}(\mathrm{p}=0.005)$ and PD-SCIC $(\mathrm{p}=0.013$; Figure 2$)$.

\section{Conclusions}

This study demonstrates the importance of correcting for surface coil-related field inhomogeneity for pixel-wise MBF quantification. However, although PD-based SCIC led to lower spatial heterogeneity than SSFP-based SCIC, the difference in measured MBF between ischaemic and remote territories was similar for both methods, and thus either could be applied. 

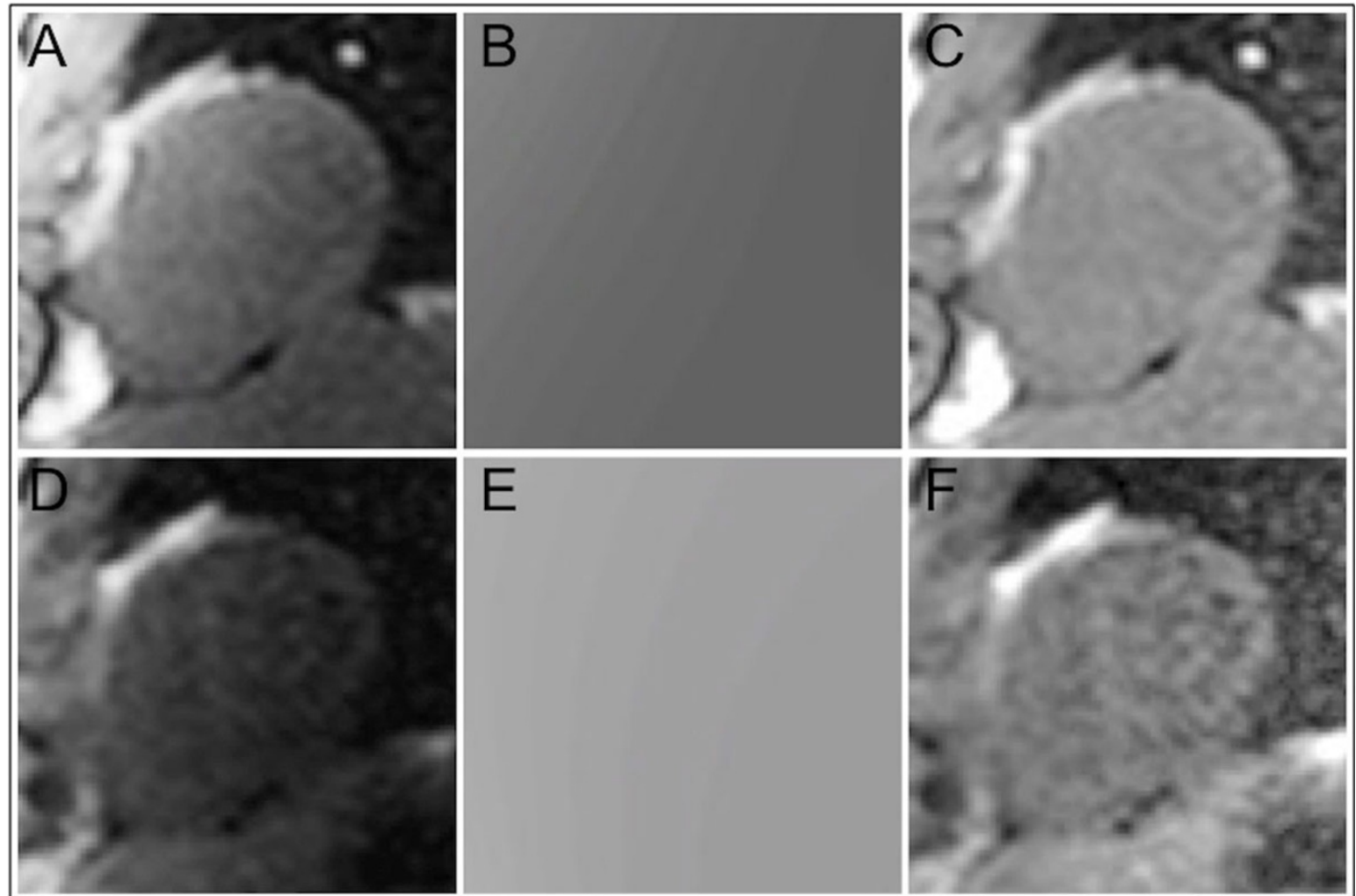

Figure 1 Surface coil intensity correction (SCIC) using proton density-weighted images (PD-SCIC; A-C) and SSFP images (SSFP-SCIC; DF). B1-field inhomogeneity was approximated using a third-order surface fit to myocardial and body signal intensities on PD (A) or SSFP (D) images in order to generate an intensity bias field ( $B$ and $E$ ), which was subsequently applied to the perfusion image series. The corrected PD and SSFP images are displayed in $\mathrm{C}$ and $\mathrm{F}$ respectively.

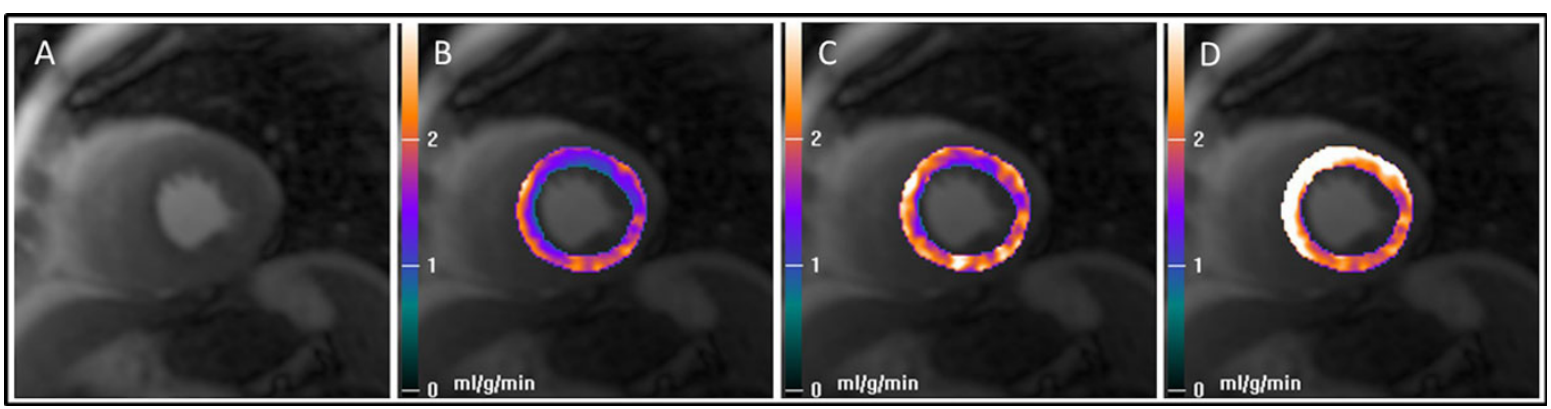

Figure 2 Mid-ventricular stress image (A) and corresponding myocardial blood flow pixel maps (B-D) in a patient with a significant stenosis in the left anterior descending (LAD) artery. The myocardial blood flow maps generated using surface coil intensity correction performed using a proton density-weighted image (PD-SCIC; B) and using a SSFP image (SSFP-SCIC; C) demonstrate a perfusion defect in the anterior wall and septum corresponding to the LAD stenosis. However, in the map generated without applying SCIC (No-SCIC; D), blood flow is highest in the anterior wall and septum, the opposite of what is correct.

\section{Funding}

CAM is supported by the National Institute for Health Research, UK. LYH, AT, HC, SW and AEA are supported by the National Institutes of Health.
doi:10.1186/1532-429X-16-S1-P364

Cite this article as: Miller et al: Quantitative pixel-wise measurement of myocardial blood flow: a comparison of methods to correct for surface coil-related field inhomogeneity. Journal of Cardiovascular Magnetic Resonance 2014 16(Suppl 1):P364. 